\title{
Endocarditis infecciosa en hospital clínico Herminda Martín de Chillán: experiencia de los últimos cinco años
}

\author{
Infective endocarditis at Herminda Martin clinical hospital of Chillan: last five years experience
}

Danitza Roa', Valentina Silva', Rocío Hernández'

\section{Resumen}

Introducción: la endocarditis infecciosa es una afección con elevada morbilidad y mortalidad, con una incidencia en Chile de 2-3 casos por 100.000 habitantes al año, con una edad de presentación en ascenso y una clínica diversa e inespecífica que requiere un manejo multidisciplinario para el manejo de estos pacientes. Materiales y métodos: estudio observacional descriptivo, se consideró el número total de fichas clínicas del hospital clínico Herminda Martín de Chillán durante los años 2015 al 2019, con diagnóstico confirmado de endocarditis infecciosa. Los datos se registraron en la hoja de recolección de datos elaborada, realizándose los análisis estadísticos pertinentes. Resultados: la muestra $(n=17)$ que pudo ser analizada tenía una edad promedio de 53,5 años; $70,5 \%$ (DE 14,50) fueron hombres y el agente más común identificado fue Staphylococcus aureus sensible a meticilina. En promedio los pacientes recibieron 28,8 días de antibióticos y la válvula más afectada fue la aórtica. Conclusiones: la endocarditis infecciosa es una patología con una gran morbimortalidad, que presenta un cuadro clínico inespecífico capaz de simular cualquier enfermedad. Se requieren aún de más estudios que reflejen la realidad nacional.

Palabras clave: endocarditis infecciosa; infección endocárdica; criterios de Duke; antibioticoterapia; microbiología: cultivos.

\begin{abstract}
Introduction: Infective endocarditis is a condition with high morbidity and mortality, with an incidence in Chile of 2-3 cases per 100,000 inhabitants per year, with increasing age of presentation and a diverse and nonspecific clinic that requires multidisciplinary management for treatment of these patients. Materials and methods: Descriptive observational study, the total number of clinical records of the Herminda Martín de Chillán clinical hospital during the years 2015 to 2019, with a confirmed diagnosis of infective endocarditis, was considered. The data were recorded in the data collection sheet prepared, performing the relevant statistical analyses. Results: The sample $(n=17)$ that could be analysed had an average age of 53.5 years (DS 14.50), 70.5\% were men, and the most common agent identified was methicillin-sensitive Staphylococcus aureus. On average, patients received 28.8 days of antibiotics, and the most affected valve was the aortic valve. Conclusions: Infective endocarditis is a pathology with high morbidity and mortality, which presents a nonspecific clinical spectrum, capable of simulating any disease. Still, more studies are required that reflect the national reality.
\end{abstract}

Keywords: Infective endocarditis; endocardial infection; Duke criteria; antibiotic therapy; microbiology; crops.

Fecha de envío: 2020-11-30 - Fecha de aceptación: 2021-03-17

\section{Introducción}

La endocarditis infecciosa es una condición potencialmente mortal con una incidencia en Chile de $2-3$ casos por 100.000 habitantes al año, siendo una afección con elevada morbilidad y mortalidad, siendo esta última de $29 \%$ en series nacionales (Flores et al., 2017) y que asciende a un $40 \%$ en series internacionales (Mohananey et al.,2018). Dentro de los cambios epidemiológicos de los últimos años se reporta una disminución de la incidencia de enfermedad reumática (Castillo et al., 2015), un aumento en la edad de presentación, siendo en promedio por encima de los 65 años. Este incremento de la enfermedad en los ancianos

(1) Medicina Interna, Universidad católica de la Santísima Concepción. Autor de Correspondencia:droa@medicina.ucsc.cl 
se debe a la mayor prevalencia de enfermedad aórtica degenerativa con o sin estenosis significativa, y al mayor acceso a procedimientos intervencionistas, utilización de prótesis cardíacas, marcapasos y catéteres intravasculares (Desimone et al., 2015). Además, se reporta mayor utilización de drogas ilícitas endovenosas, que constituye un importante factor de riesgo para la generación de la patología. Por otro lado, se ha evidenciado mejora en los métodos diagnósticos, lo cual ha sido fundamental al momento de otorgar tratamiento precoz (Flores et al., 2017). La microbiología de la enfermedad también ha cambiado, y los estafilococos, más a menudo asociados con el contacto médico y los procedimientos invasivos, han superado a los estreptococos como la causa más común de la enfermedad (Cahill \& Prendergast, 2016).

La presentación clínica de la endocarditis infecciosa es particularmente diversa e inespecífica. Esta patología debe ser considerada en cualquier persona con sepsis de origen desconocido o fiebre en presencia de factores de riesgo. Las manifestaciones de la sepsis pueden variar del malestar general al shock, influenciado por la virulencia microbiana y la respuesta inmune del huésped (Werdan et al., 2014). La endocarditis también puede presentarse como una complicación, particularmente accidente cerebrovascular o embolia sistémica. La evaluación clínica inicial de un paciente con sospecha de endocarditis infecciosa implica la valoración de factores de riesgo y una búsqueda de antecedentes y hallazgos en el examen físico que apoyen el diagnóstico. Dentro de los factores de riesgo, se describen episodios previos de endocarditis, una válvula protésica o un dispositivo cardíaco, y cardiopatía valvular o congénita. También el uso de drogas intravenosas, vías centrales por tiempo prolongado, inmunosupresión, un procedimiento quirúrgico dental reciente, entre otros (Cahill \& Prendergast, 2016). El diagnóstico de endocarditis infecciosa requiere la integración de hallazgos clínicos, análisis microbiológicos y resultados de imágenes, siendo de utilidad los Criterios de Duke (Tabla 1) con una sensibilidad y especificidad de aproximadamente el $80 \%$ (Durack et al., 1994), sin embargo, no reemplazan el juicio clínico. Su precisión diagnóstica es buena en patología de válvula nativa izquierda, pero decae en presencia de endocarditis de válvulas derechas, válvula protésica, de marcapasos o con hemocultivos negativo (Casabé et al.,2016). En cuanto al tratamiento, requiere de un equipo multidisciplinario para brindar el manejo a estos pacientes. Por las razones anteriormente expuestas se establece como objetivo de la investigación, contribuir al conocimiento médico en relación a las características epidemiológicas de los pacientes diagnosticados con Endocarditis Infecciosa entre el periodo transcurrido entre el año 2015 y 2019 en el Hospital Clínico Herminda Martin de Chillán.

\section{Materiales y métodos}

Para el desarrollo de la investigación, se realizó un estudio observacional descriptivo, utilizando una metodología cuantitativa, para ello se consideró el universo de pacientes diagnosticados con endocarditis infecciosa no especificada y endocarditis aguda y subaguda en el hospital clínico Herminda Martin de Chillán durante los años 2015 al 2019 (clasificados por CIE10 I33.O), teniendo así un total de 25 pacientes. Se excluyeron del análisis descriptivo, 8 pacientes por no contar con los datos necesarios mínimos, ya que no se pudo acceder a su ficha clínica. Quedando una población total de 17 pacientes.

Recolección de datos: Se realizó revisión de las fichas clínicas de los pacientes descritos, durante los meses de julio-agosto del año 2020, registrándose las siguientes variables: sexo, edad, síntomas, antecedentes, hallazgos en hemocultivo y ecocardiograma, tratamiento antibiótico recibido y duración de este. La elección de las variables, se debe a la relevancia de estos datos. Cabe precisar que de las variables escogidas no se encuentran datos personales ni sensibles de los pacientes ya que se procuró el resguardo de la identidad e información personal del grupo en estudio.

\section{Análisis estadístico:}

Se trabajó mediante la tabulación de datos estadísticos clínicos y epidemiológicos a través del software de hojas de cálculo, Microsoft Excel, los datos fueron procesados en cuanto a frecuencias porcentuales y desviación estándar.

\section{Consideraciones éticas:}

El protocolo fue revisado y aprobado por el comité de ética del hospital clínico Herminda Martín de Chillán durante el mes de marzo del 2020. Para la realización de este proyecto no fue necesario el uso de consentimiento informado.

\section{Resultados}

En cuanto a las características de la población de la muestra seleccionada $n=17$, el promedio de edad fue de 53,52 años (DE 14.50), con un rango etario de 26 a 76 años; 70,59\% $(n=12)$ correspondió a hombres (Tabla 2).

Por otra parte, considerando las características de la enfermedad (Tabla 3) el 100\% ( $n=17)$ de los pacientes presentó malestar general; $76,47 \%(n=13)$ cansancio y el $88,23 \%(n=15)$ fiebre. Un paciente presentó fenómenos vasculares: embolia al sistema nervioso central, manifestándose como un accidente cerebro vascular isquémico. El $58,82 \%(n=10)$ de los pacientes contaba con cardiopatías predisponentes para el desarrollo de endocarditis bacteriana. 76,47\% $(n=13)$ se presentó con un hemocultivo positivo al momento del 
Roa et al.

diagnóstico. No se registraron fenómenos inmunológicos en ninguno de los pacientes.

El agente etiológico más aislado fue Staphylococcus aureus sensible a meticilina ( $n=4)$ (Tabla 4). El resto de los agentes etiológicos fueron: Streptococcus pneumoniae, Streptococcus gallolitycus, Streptococcus sanguinis, Staphylococcus aureus resistente a meticilina y
Streptococcus viridians entre otros. En promedio los pacientes recibieron 28,88 días de tratamiento con antibióticos (DE 10.98)

El hallazgo más frecuente en la ecografía fue la presencia de vegetaciones (76,47\%; $n=13)$ (Tabla 5), luego la presencia de insuficiencia valvular (64,71\%; n=11). La válvula más afectada fue la aórtica en un $58,82 \%$ de los casos $(n=10)$.

Tabla 1: Criterios de Duke.

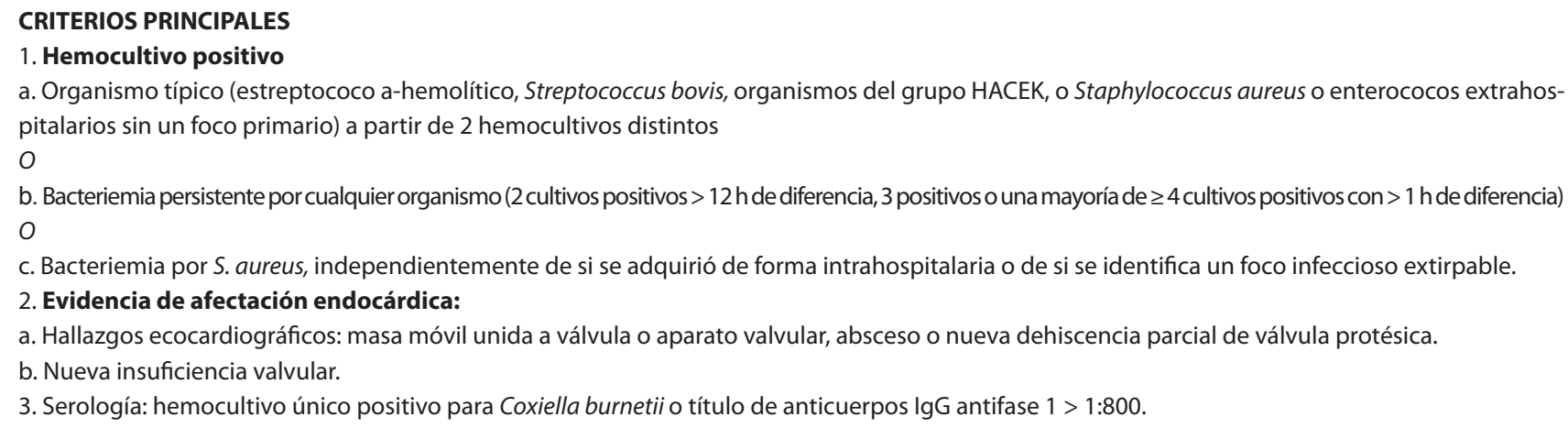

\section{CRITERIOS MENORES}

1. Condición predisponente: consumo de drogas intravenosas o alteración cardíaco predisponente.

2. Fiebre $\geq 38^{\circ} \mathrm{C}$.

3. Fenómenos vasculares: embolia arterial, émbolos pulmonares sépticos, aneurisma micótico, hemorragia intracraneal, hemorragias conjuntivales, lesiones de Janeway.

4. Fenómenos inmunitarios: glomerulonefritis, nódulos de Osler, manchas de Roth, factor reumatoide.

5. Hallazgos ecocardiográficos acordes con endocarditis pero que no cumplen los criterios principales.

6. Evidencia microbiológica: hemocultivos positivos que no cumplen los criterios principales o evidencia serológica de infección activa acorde con endocarditis.

\section{ENDOCARDITIS INFECCIOSA DEFINITIVA}

1. Endocarditis infecciosa demostrada anatomopatológicamente

O

2. Criterios clínicos que cumplen

a. Dos criterios principales o

b. Un criterio principal y uno menor $o$

c. Tres criterios menores

\section{ENDOCARDITIS INFECCIOSA POSIBLE}

Hallazgos que no satisfacen los criterios de endocarditis infecciosa definitiva pero no permiten rechazarla

\section{ENDOCARDITIS INFECCIOSA RECHAZADA}

1. Diagnóstico alternativo o

2. Resolución de síndrome de endocarditis infecciosa con tratamiento antibiótico durante $\leq 4$ días o

3. Ausencia de evidencia anatomopatológica de endocarditis infecciosa en cirugía o autopsia con tratamiento antibiótico durante $\leq 4$ días.

HACEK = especies de Haemophilus y Aggregatibacter (antes Actinobacillus actinomycetemcomitans), Cardiobacterium hominis, Eikenella corrodens y especies de Kingella; $\lg \mathrm{G}=$ inmunoglobulina $\mathrm{G}$.

Adaptado de Li JS et al. (2000). 
Roa et al.

Tabla 2: Características de la población.

\begin{tabular}{l|c|c}
\hline Variable & Promedio & DE \\
\hline Edad (años) & 53,52 & 14,50 \\
\hline Características & $\mathrm{n}$ & $\%$ \\
\hline Sexo masculino (\%) & 15 & 70,59 \\
\hline Cardiopatias predisponentes & 10 & $58,82 \%$ \\
\hline Válvula protésica & 6 & 35,39 \\
\hline Enfermedad reumática en válvula nativa & 3 & 17,64 \\
\hline Dispositivo automático implantable & 1 & 5,88
\end{tabular}

Tabla 3: Características de la enfermedad.

\begin{tabular}{l|c|c}
\hline Síntomas y signos & $\mathbf{n}$ & $\%$ \\
\hline Fiebre (\%) & 15 & 88,23 \\
\hline Sudoración nocturna (\%) & 4 & 23,53 \\
\hline Cansancio (\%) & 13 & 76,47 \\
\hline Malestar general (\%) & 17 & 100 \\
\hline Pérdida de peso (\%) & 4 & 23,52 \\
\hline Vasculares de Duke (\%) & 1 & 5,88 \\
\hline Fenómenos inmunológicos (\%) & 0 & 0 \\
\hline Cardiopatías predisponentes & 10 & 58,82 \\
\hline Hemocultivo positivo & 13 & 76,47 \\
\hline
\end{tabular}

Tabla 4: Características microbiológicas.

\begin{tabular}{|c|c|c|c|c|}
\hline Caso & Edad/Sexo & Agente Etiológico & Tratamiento antibiótico recibido & Total de días \\
\hline 1 & $51 / \mathrm{M}$ & - & Vancomicina y amikacina & 63 \\
\hline 2 & $32 / \mathrm{M}$ & Streptococcus pneumoniae & Ceftriaxona & 21 \\
\hline 3 & $63 / F$ & Staphylococcus aureus sensible a meticilina & Cloxacilina & 15 \\
\hline 4 & $26 / \mathrm{M}$ & - & Vancomicina meropenem & 28 \\
\hline 5 & $47 / \mathrm{M}$ & Streptococcus gallolitycus & Ceftriaxona gentamicina & 28 \\
\hline 6 & $38 / F$ & Streptococcus sanguinis & Vancomicina, gentamicina, rifampicina / ceftriaxona & 28 \\
\hline 7 & $26 / \mathrm{M}$ & Staphylococcus aureus resistente a meticilina & Vancomicina & 42 \\
\hline 8 & $65 / \mathrm{M}$ & Streptococcus viridians & Penicilina y gentamicina & 24 \\
\hline 9 & 75/M & - & Cloxacilina y penicilina sódica & 35 \\
\hline 10 & $70 / \mathrm{M}$ & Staphylococcus aureus sensible a meticilina & Ceftriaxona ampicilina sulbactam & 52 \\
\hline 11 & $34 / F$ & - & Meropenem, vancomicina & - \\
\hline 12 & $76 / \mathrm{F}$ & Streptococcus grupo C multiresistente & Ceftriaxona & 21 \\
\hline 13 & $61 / \mathrm{M}$ & Streptococcus agalactiae & Penicilina, ciprofloxacino & 23 \\
\hline 14 & $74 / \mathrm{M}$ & Staphylococcus aureus sensible a meticilina & Cloxacilina & 13 \\
\hline 15 & $58 / \mathrm{F}$ & Enterococo faecalis & Amikacina & 15 \\
\hline 16 & $63 / M$ & Staphylococcus aureus sensible a meticilina & Cloxacilina & 7 \\
\hline 17 & $51 / \mathrm{M}$ & Staphylococcus aureus resistente a meticilina & Cloxacilina & 15 \\
\hline & & & $\begin{array}{l}\text { Promedio } \\
\text { (Desviación estándar) }\end{array}$ & $\begin{array}{l}28,88 \\
10,98\end{array}$ \\
\hline
\end{tabular}


Roa et al.

Tabla 5: Características generales eco cardiográficas.

\begin{tabular}{|c|c|c|c|c|c|}
\hline Caso & Edad/Sexo & Vegetaciones & Absceso & Insuficiencia valvular & Válvula afectada \\
\hline 1 & $51 / \mathrm{M}$ & 1 & 0 & 1 & Aórtica \\
\hline 2 & $32 / \mathrm{M}$ & 1 & 1 & 1 & Aórtica \\
\hline 3 & $63 / F$ & 1 & 0 & 1 & Aórtica \\
\hline 4 & $26 / M$ & 1 & 0 & 1 & Mitral y Aórtica \\
\hline 5 & $47 / M$ & 1 & 0 & 1 & Aórtica \\
\hline 6 & $38 / F$ & 1 & 1 & 0 & Aórtica \\
\hline 7 & $26 / M$ & 1 & 0 & 0 & Tricúspide \\
\hline 8 & $65 / M$ & 1 & 1 & 1 & Mitral \\
\hline 9 & 75/M & 1 & 1 & 1 & Mitral \\
\hline 10 & $70 / \mathrm{M}$ & 0 & 0 & 1 & Mitral y aortica \\
\hline 11 & $34 / F$ & 0 & 0 & 1 & Mitral \\
\hline 12 & $76 / F$ & 1 & 0 & 0 & Mitral y aortica \\
\hline 13 & $61 / \mathrm{M}$ & 0 & 0 & 1 & Mitral \\
\hline 14 & $74 / \mathrm{M}$ & 1 & 0 & 0 & Mitral \\
\hline 15 & $58 / F$ & 1 & 0 & 0 & Aortica \\
\hline 16 & $63 / \mathrm{M}$ & 1 & 0 & 0 & Aortica \\
\hline 17 & $51 / \mathrm{M}$ & 0 & 0 & 1 & Mitral \\
\hline
\end{tabular}

\section{Discusión}

En este estudio se observó una mayor afección de endocarditis infecciosa en el sexo masculino, lo que concuerda con varios estudios tanto a nivel nacional (Merello et al., 2019) como internacional (Kazelian et al.,2012; Cecil, 2013; Torres et al.,2018).La edad media de presentación de la muestra analizada se encontraba alrededor de la quinta década de la vida, tal y como se señala en la literatura (Merello et al., 2019), lo que se encuentra en estrecha relación con el aumento de la sobrevida poblacional (Albala, 2019), lo que trae consigo un aumento de comorbilidades predisponentes, como lo es la cardiopatía degenerativa y estados de inmunosupresión como la enfermedad renal crónica y la diabetes mellitus (Flores et al., 2017). Por otra parte en esta serie, la estancia hospitalaria fue de 31 días en promedio, lo que se ve influenciado por múltiples variables como lo es la duración del tratamiento antibiótico, la necesidad de cirugía y la presencia de complicaciones aguda, además de la necesidad de un equipo multidisciplinario para el adecuado manejo, dado que, a pesar que existen los lineamientos generales para el tratamiento de la endocarditis infecciosa, ninguna de las recomendaciones en lo que respecta a directrices internacionales sobre esta patología, están respaldados por un nivel de evidencia tipo A (Cahill \& Prendergast, 2016).

En cuanto a la presentación clínica de la endocarditis infecciosa ésta es sumamente diversa e inespecífica, describiéndose ya por Osler (1885), como una de las enfermedades con mayores dificultades diagnósticas, hecho que ha sido mantenido por varios autores (Sénior \& Gándara, 2015; Cahill \& Prendergast, 2016), 130 años más tarde y que es coincidente con esta serie dada la gran variedad de síntomas de los pacientes estudiados en la muestra, siendo frecuente el malestar general, el cansancio y la fiebre como presentación clínica inicial del cuadro, al igual como se describen en otras cohortes (Sénior \& Gándara, 2015), la pérdida de peso y sudoración nocturna estuvieron presentes pero en una menor proporción. En cuanto a los fenómenos vasculares el 5,88\% de la muestra presentaba algún tipo de fenómeno embólico, cifra un tanto menor al compararlo con otras series (Torres et al.,2018), habiendo ausencia de fenómenos inmunológicos como manifestación clínica en la muestra analizada. Todo esto nos lleva a reforzar la idea ya antes planteada, en que la endocarditis infecciosa se constituye como un cuadro clínico inespecífico capaz de simular cualquier enfermedad, que nos puede llevar a errar a la hora del diagnóstico clínico, por lo que recomendamos tener un alto índice de sospecha tanto en médicos generales como especialistas al momento de enfrentar pacientes que cuentan con factores de riesgo, como lo es el antecedente de endocarditis previa, presencia de válvula protésica o un dispositivo cardíaco, cardiopatía valvular o congénita, inmunosupresión o extracción dental reciente (Cahill \& Prendergast, 2016).

El antecedente de algún tipo de cardiopatía como factor predisponente para el desarrollo de endocarditis infecciosa ha sido ampliamente descrito por la literatura (Cahill \& Prendergast, 2016; Harrison, 2018), sin embargo, Castillo et al. (2002) señala que la tasa de endocarditis infecciosa en pacientes sin cardiopatía predisponente tiende a ser más alta en el último tiempo, lo que se justifica en parte por la disminución en la incidencia de la enfermedad valvular reumática y la mayor frecuencia de factores de riesgo asociados, como la diabetes mellitus, la enfermedad inflamatoria 
intestinal crónica o la insuficiencia renal crónica, a pesar de lo anterior, en esta serie la presencia de cardiopatía predisponente se encontró en un 58,8\% los casos y si bien el antecedente de enfermedad reumática no fue prevalente en esta cohorte, varios de los pacientes analizados contaban con prótesis valvulares y dispositivos electrónicos implantables, lo que podría explicar este mayor porcentaje.

El hemocultivo se encontraba positivo en un 76,4\% de los casos, cultivo que resulta fundamental tanto para el diagnóstico como para determinar la sensibilidad antimicrobiana que permitirá definir el tratamiento, el 23,6\% restante de cultivos se encontrada negativo en esta serie, cifras muy similares a las expuesta en el estudio de Merello et al.(2019), realizado con 103 pacientes que reportan un $26 \%$ de cultivos negativos, lo que se contrapone a cifras reportadas en estudios internacionales realizados en países de Europa donde los cultivos negativos no superan el 20\% (Castillo et al., 2002; Lamas \& Eykyn, 2003; Saito et al., 2014), estas tasas de negatividad reportadas pueden ser explicadas por el inicio precoz de antibióticos, por una mala realización de la técnica o bien por una alta prevalencia de microorganismos que no crecen en los cultivos habituales. De los cultivos positivos, las bacterias más prevalentes fueron Staphylococcus y Streptococcus, con una predominancia del primero por sobre el segundo, lo que concuerda con lo reportado en la literatura actual (Torres et al.,2018) donde se ha visto un incremento del Staphylococcus aureus como el patógeno más frecuentemente aislado en los hemocultivos, hecho que además ha sido evidenciado en el estudio nacional de Flores et al. (2017).

La ecocardiografía ha sido descrita como el proceder que sigue en orden de importancia a toma de hemocultivos (Torres et al., 2018), ya que permite precisar vegetaciones, su localización, tamaño y movilidad, además de ser útil para el reconocimiento de las cardiopatías subyacentes en caso de que existan y precisa el estado de la función ventricular, bajo este concepto el $76,4 \%$ de la muestra tuvo vegetaciones al examen imagenológico, viéndose afectada las válvulas del corazón izquierdo en un 94\%, cifra que está por sobre lo publicado en otras series, donde la infección del hemicardio izquierdo se reporta hasta un $71 \%$ de los casos (Castillo et al., 2002), por lo que el seguimiento de estos pacientes y la evaluación constante debe ser primordial ya que como se señala en la literatura, aproximadamente $70 \%$ de los pacientes con endocarditis izquierda va a requerir cirugía, de los cuales $48 \%$ finalmente se opera (Merello et al., 2019), asociándose esto a múltiples complicaciones y aumento de la mortalidad postoperatoria (Patrat-Delon et al., 2016; Fernández et al., 2018).

\section{Conclusiones}

A partir de este análisis se puede concluir que, si bien existen estudios actuales a nivel nacional respecto al tema, no contamos con estudios que abarquen un gran número poblacional que permitan reflejar la realidad nacional de esta patología, cuya importancia radica en la gran morbimortalidad y gasto sanitario que trae consigo.

\section{Reconocimientos}

No contamos con asistencia financiera, técnica y / o editorial durante la realización de este trabajo. Tampoco tenemos conflicto de interés.

\section{Referencias}

Albala C. (2019). El envejecimiento de la población chilena y los desafíos para la salud y el bienestar de las personas mayores. Revista Médica Clínica Las Condes 31, 7-12.

Cahill J. \& Prendergast, B. (2016). Infective endocarditis. The Lancet 387(10021), 882-893.

Casabé J, Giunta G, Barisani J. \& Nacinovich F. (2016). Consenso de Endocarditis Infecciosa. Revista Argentina de Cardiología 84, 9. Castillo FJ, Anguita M, Castillo JC, Ruiz M, Mesa D. \& Suarez de Lezo J. (2015). Changes in Clinical Profile, Epidemiology and Prognosis of Left-sided Native-valve Infective Endocarditis Without Predisposing Heart Conditions. Revista Española Cardiología (Engl Ed) 68, 445.

Castillo FJ, Anguita M, Torres F, Siles J, Mesa D. \& Vallés F. (2002). Risk Factors Associated with Endocarditis without Underlying Heart Disease. Revista Española de Cardiología (Engl Ed) 55, 304-307.

Cecil. (2013). Tratado de Medicina Interna. Elsevier España 76, 466-476.

Desimone D, Desimone C, Tleyjeh I, Correa D, Anakevar D. \& Lahr B. (2015) Association of Mitral Valve Prolapse With Infective Endocarditis Due to Viridans Group Streptococci. Clinical Infectious Diseases 61, 623.

Durack DT, Lukes AS. \& Bright DK. (1994). New criteria for diagnosis of infective endocarditis: utilization of specific echocardiographics findings. Duke Endocarditis Service. The American Journal of Medicine 96, 200-9.

Fernández-Hidalgo N, Ferrería-González I, Marsal JR, Ribera A, Aznar ML. \& De Alarcón A. (2018). A pragmatic approach for mortality prediction after surgery in infective endocarditis: optimizing and refining EuroSCORE. Clinical Microbiology and Infection 24, 1102 e7-e15. 
Roa et al.

Flores P, González N, Betancourt P, Berho J, Astudillo C. \& García C. (2017). Endocarditis Infecciosa: caracterización clínica de la enfermedad. Revisión de casos de los últimos 5 años. Revista Chilena Cardiología 36, 34-40.

Harrison. (2018). Principios de Medicina Interna. Mc Graw Hill 118, 789-798.

Kazelian L, Vidal L, Neme R. \& Gagliardi J. (2012). Endocarditis infecciosa activa: 152 casos. Revista Medicina 72, 109-114.

Lamas CC. \& Eykyn SJ. (2003). Blood culture negative endocarditis: analysis of 63 cases presenting over 25 years. Heart 89, 258-62.

Merello L, Salazar M, Elgueta F, González D, Elton V. \& Quiroz M. (2019) Sobrevida a 10 años de pacientes egresados luego de cirugía por endocarditis infecciosa en un hospital público. Revista médica de Chile 147, 1535-1542.

Mohananey D, Mohadjer A. \& Pettersson G. (2018). Association of Vegetation Size With Embolic Risk in Patients With Infective Endocarditis: A Systematic Review and Meta-analysis. JAMA Internal Medicine 178, 502-510.

Osler W. (1885). The Gulstonian Lectures, on Malignant Endocarditis. The British Medical Journal 1, 577-79
Oyonarte M, Montagna R, Braun S, Rojo P, Jara J, Cereceda M, Morales M, Nazal C. \& Alonso F. (2012). Endocarditis infecciosa: características clínicas, complicaciones y mortalidad en 506 pacientes y factores pronósticos de sobrevida a 10 años (1998-2008). Estudio cooperativo nacional en endocarditis infecciosa en Chile (ECNEI-2). Revista médica de Chile 140.

Patrat-Delon S, Rouxel A, Gacouin A, Revest M, Flecher E. \& Fouquet O. (2016). EuroSCORE II underestimates mortality after cardiac surgery for infective endocarditis. European Journal of Cardiothoracic Surgery 49, 944-51.

Saito MPC, Valle A. \& Castañeda E. (2014). Tratamiento quirúrgico de la endocarditis infecciosa en un hospital general: Indicaciones y morbimortalidad. Revista Médica Herediana 25,135-41.

Sénior J. \& Gándara R. (2015). Endocarditis infecciosa. latreia 28, 456-471.

Torres J, Sánchez I, Peña H, Pérez C \& Pérez E. (2018). Caracterización clínica de pacientes con endocarditis. Revista Electrónica Dr. Zoilo E. Marinello Vidaurreta 43.

Werdan K, Dietz S. \& Löffl er B. (2014). Mechanisms of infective endocarditis: pathogen-host interaction and risk states. Nature Reviews Cardiology 11, 35-50. 